\title{
Hybrid placement technique for hepatic hilar obstruction using a new uncovered self-expandable metal stent $\square$
}

\section{(ㄷ)(1) $\ominus$}

\author{
Authors \\ Institutions \\ 1 Premier Development Research of Medicine, Osaka \\ Medical College, Osaka, Japan \\ 2 2nd Department of Internal Medicine, Osaka Medical \\ College, Osaka, Japan \\ submitted 11.4.2019 \\ accepted after revision $\quad$ 17.6.2019 \\ Bibliography \\ DOI https://doi.org/10.1055/a-0998-7847 | \\ Endoscopy International Open 2019; 07: E1288-E1292 \\ (c) Georg Thieme Verlag KG Stuttgart · New York \\ elSSN 2196-9736 \\ Corresponding author \\ Takeshi Ogura, Premier Development Research of \\ Medicine, 2nd Department of Internal Medicine, Osaka \\ Medical College, 2-7 Daigakuchou, Takatsukishi, Osaka \\ 569-8686, Japan \\ Fax: $+81-726846532$ \\ oguratakeshi0411@yahoo.co.jp
}

Takeshi Ogura ${ }^{1,2}$, Masanori Yamada², Saori Ueno ${ }^{2}$, Shinya Fukunishi ${ }^{1,2}$, Kazuhide Higuchi $^{2}$

\section{ABSTRACT}

Background and study aim Recently, a novel technique combining stent-in-stent (SIS) and side-by-side (SBS) technique (hybrid technique) has been reported. This technique may have application for hepatic hilar obstruction (HBO) because it reduces the number of times the stent mesh has to be passed. However, to the best our knowledge, evaluation of its use in that setting has not been reported. Recently, a new uncovered self-expandable metal stent (UCSEMS) has become available in Japan. The diameter of this stent delivery shaft is only $5.4 \mathrm{Fr}$, and the discrepancy between the guidewire and top of stent delivery system is strongly reduced. We herein report technical tips for hybrid metal stent deployment for high-grade HBO using this novel UCSEMS.

Hybrid metal stent deployment using he novel UCSEMS was attempted in seven patients. Technical success was obtained in all patients. Mean procedure time was 33.3 minutes (range, 27 to 43). Clinical success was obtained in six patients. Median stent patency was 109 days (range, 48-160 days).

In conclusion, the hybrid metal stent placement technique appears to be useful using the new UCSEMS because of its fine-gauge stent delivery system.

\section{Introduction}

Biliary drainage under endoscopic retrograde cholangiopancreatography (ERCP) guidance is the gold standard technique for bile duct obstruction. In patients who have multiple biliary obstructions and complications such as hepatic hilar obstruction (HBO), deployment of multiple stents is needed. A recent randomized trial showed that bilateral biliary drainage resulted in fewer reinterventions and favorable stent patency compared with unilateral biliary drainage [1]. For bilateral stent deployment, use of both a side-by-side (SBS) and a stent-in-stent (SIS) technique have been reported [2-6]. In the case of highgrade $\mathrm{HBO}$, which sometimes requires placement of three or more stents, the SIS technique may be challenging. The SBS technique is also challenging because of the limited diameter of the common bile duct. Recently, a novel technique combining SIS and SBS (hybrid technique) has been reported for such cases [7]. This novel technique may have applications for $\mathrm{HBO}$ because it reduces the number of times the stent mesh has to be passed. Recently, a new UCSEMS has become available in Japan. We herein report technical tips for hybrid metal stent deployment for high-grade HBO using this novel UCSEMS in a pilot study.

\section{Patients and methods}

Patients with complicated HBO were enrolled between November 2018 and January 2019. According to the Bismuth-Corlette classification, only patients with consecutive type Illa were included. All patients were histologically diagnosed as having unresectable $\mathrm{HBO}$ using endoscopic ultrasound-guided fine-needle aspiration.

For this study protocol, bilateral stent deployment in the left and anterior bile ducts were performed first If infected bile 

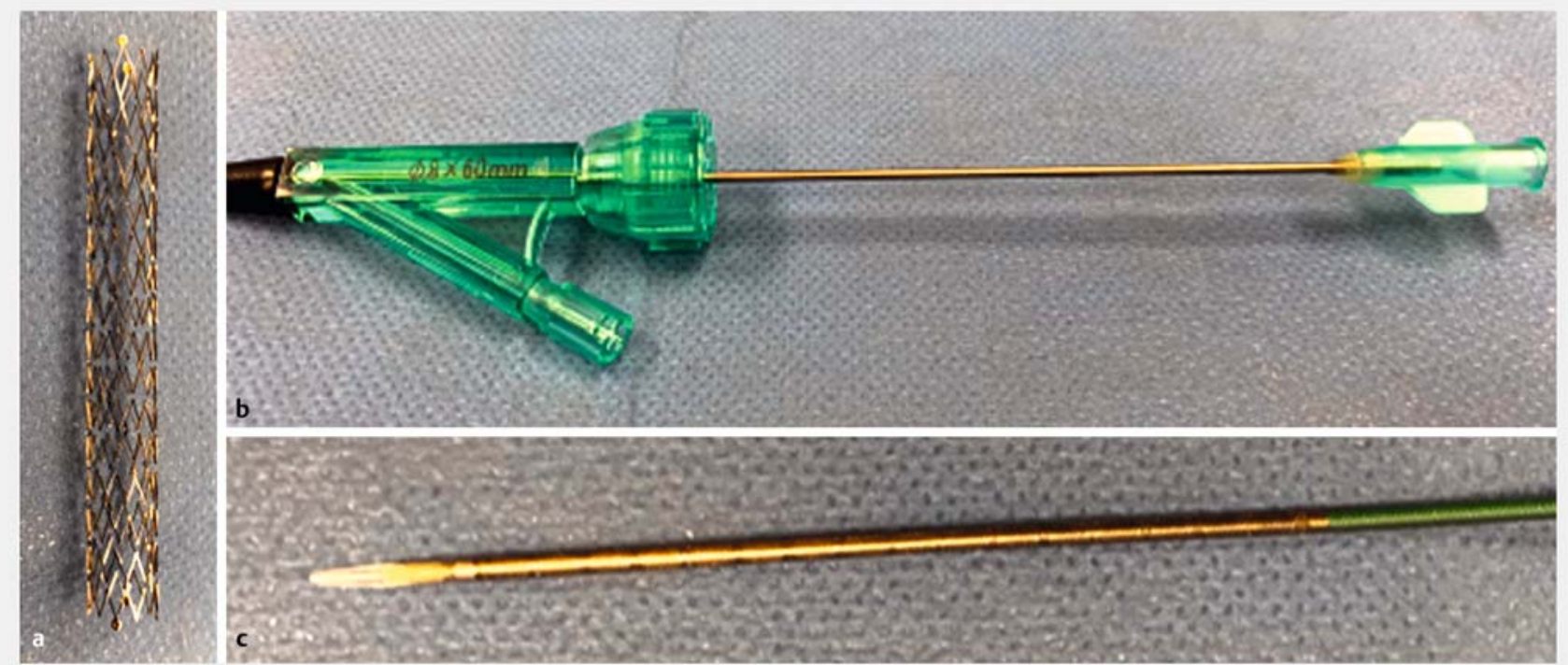

- Fig. 1 A newly UCSEMS (ZEOSTENT V: Zeon Medical Inc., Tokyo, Japan). a This stent is laser cutting type UCSEMS. b The stent delivery system is only $5.4 \mathrm{Fr}$, and $\mathbf{c}$ discrepancy between the guidewire and top of stent delivery system is strongly reduced.

juice was aspirated from the posterior bile duct, deployment of triple stents was attempted. In this study, patients who underwent triple stent deployment using the hybrid technique were enrolled.

This study was approved by the ethics committee of our hospital, and patients provided written, informed consent for all procedures associated with it.

\section{Technical tips for hybrid metal stent deployment}

- Fig. 1 shows the new UCSEMS (ZEOSTENT V: Zeon Medical Inc., Tokyo, Japan). This stent is a laser-cutting type UCSEMS ( Fig.1a), available in diameters from $6 \mathrm{~mm}$ to $10 \mathrm{~mm}$ and lengths from $6 \mathrm{~cm}$ to $10 \mathrm{~cm}$. The stent delivery system is $5.4 \mathrm{Fr}$ ( $\vee$ Fig. 1b), and the discrepancy between the guidewire and the top of the stent delivery system is strongly reduced ( $\bullet$ Fig. $1 \mathrm{c}$ ). Therefore, stent insertion into the biliary tract across the stricture site or through the mesh technique may be easy.

- Video 1 shows technical tips for the hybrid metal stent deployment technique. A JF260V duodenoscope (Olympus Optical, Tokyo, Japan) was advanced into the ampulla of Vater, followed by biliary cannulation with a standard ERCP catheter (MTW Endoskopie, Düsseldorf, Germany). If the main pancreatic duct was to be cannulated, a 0.025 -inch guidewire was inserted for the double guidewire technique. After achieving biliary cannulation, contrast medium was injected into the biliary tract, and the site of obstruction was evaluated. Frist, the 0.025-inch guidewire (VisiGlide 1; Olympus Medical Systems, Tokyo, Japan) was deployed into the left, anterior, and posterior bile ducts. During this procedure, the ERCP catheter was inserted into the posterior bile duct and bile juice was aspirated. If the bile juice was infected, deployment of triple stents was attempted. Then, stent delivery systems were simultaneously inserted into the left and right bile ducts using two devices in a one-channel method ( $\triangleright$ Fig. 2a) and stent deployment was simultaneously performed. After the guidewire was removed from the left bile duct, the guidewire in the posterior bile duct was advanced into the anterior bile duct through the mesh of the UCSEMS in the posterior bile duct ( $\triangleright$ Fig. $\mathbf{2 b}$ ). Next, the stent delivery system was inserted into the anterior bile duct over the guidewire ( $\bullet$ Fig. $2 \mathbf{c}$ ), and stent deployment

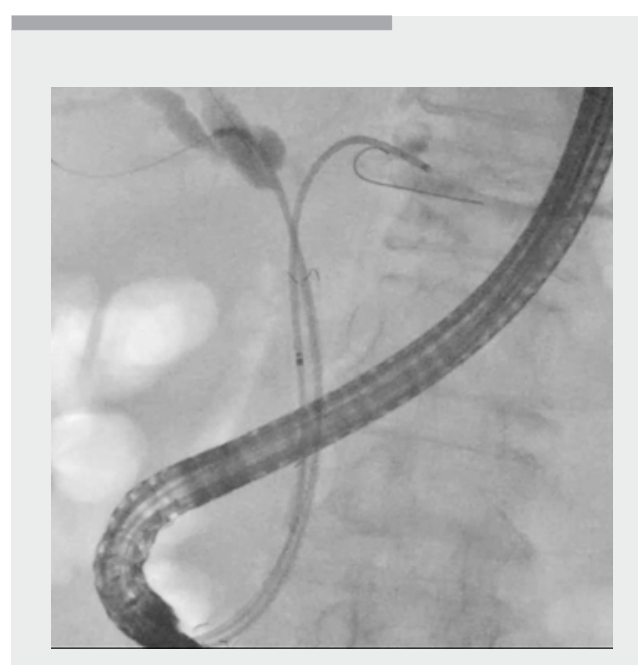

$\nabla$ Video 1 According to cholangiography, high-grade hepatic hilar obstruction is seen. Guidewire deployments are performed in the left, anterior, and posterior bile duct. Then, newly uncovered self-expandable metal stent deployments are simultaneously performed in the left and posterior bile ducts. Finally, after the guidewire is inserted into the anterior bile duct through the mesh of metal stent in the posterior bile duct, stent deployment is performed in the anterior bile duct. 

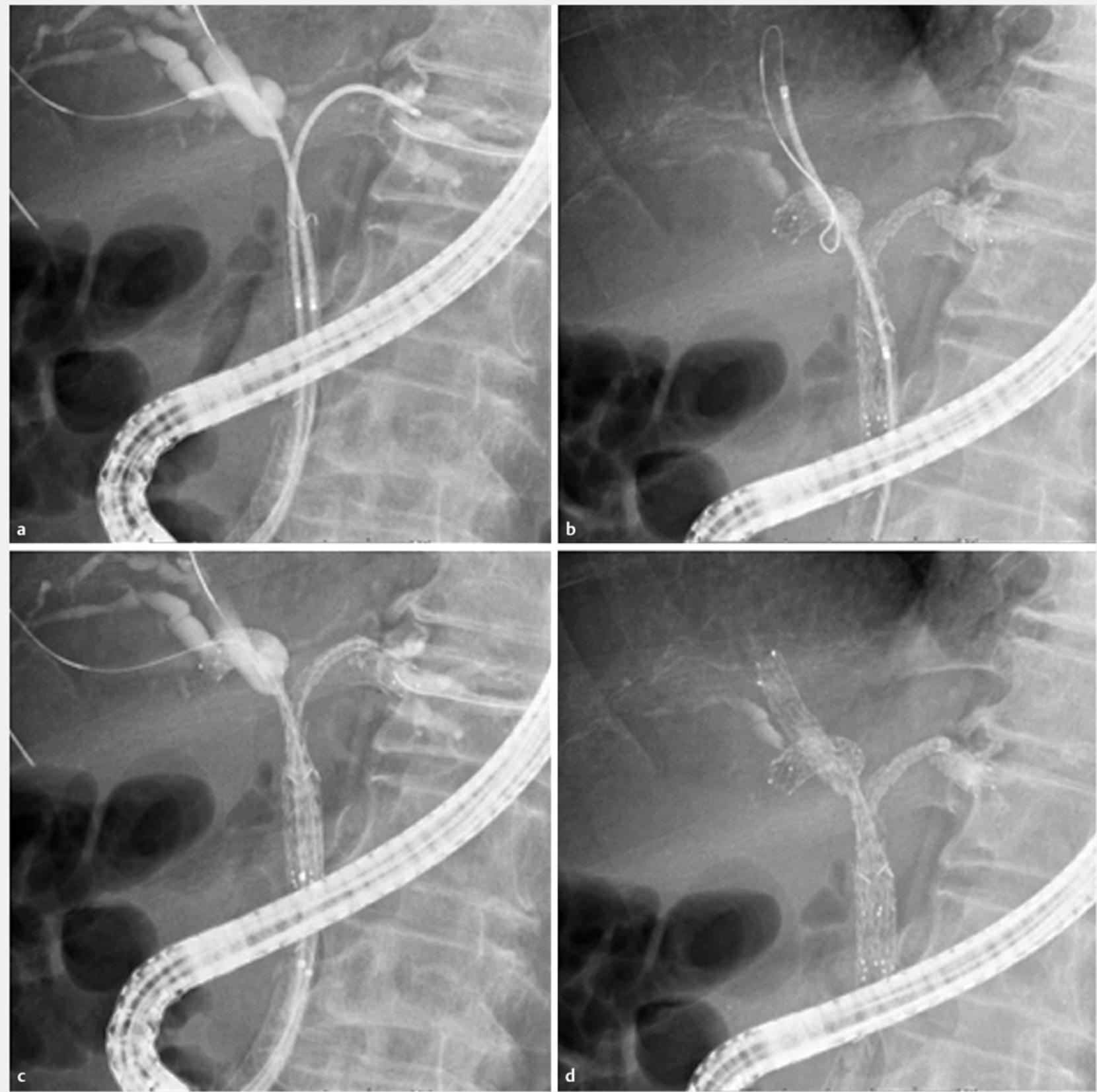

- Fig. 2 a Stent delivery systems is inserted into the left and posterior bile ducts using two devices in one channel method. b Stent deployments are simultaneously performed. c The stent delivery system is inserted into the anterior bile duct over the guidewire. $\mathbf{d}$ Stent deployment is successfully performed.

was successfully performed without any adverse events (AE) ( Fig.2d).

\section{Definitions}

Technical success was defined as deployment of three stents using hybrid metal stent deployment in a single session. Clinical success was defined as a reduction in bilirubin values to normal $(<1.3 \mathrm{mg} / \mathrm{dL})$ or less than half of the pre-drainage value within 1e month. Procedural duration was defined as the amount of elapsed time between scope insertion and stent deployment.
Stent dysfunction was determined based on recurrent obstructive jaundice, clinical symptoms suggestive of acute cholangitis, and dilation of the biliary tracts on computed tomography. Stent patency was measured from stent deployment to stent dysfunction or the patient's death. AEs were graded according to the severity grading system of the American Society for Gastrointestinal Endoscopy lexicon [8]. 
- Table 1 Patient characteristics.

\begin{tabular}{|c|c|c|c|c|c|c|c|c|c|c|}
\hline No & $\begin{array}{l}\text { Age/ } \\
\text { gender }\end{array}$ & $\begin{array}{l}\text { Diagnosis } \\
\text { of } \mathrm{HBO}\end{array}$ & $\begin{array}{l}\text { Reason for } \\
\text { unresectability }\end{array}$ & $\begin{array}{l}\text { Chemo- } \\
\text { therapy }\end{array}$ & $\begin{array}{l}\text { Technical } \\
\text { success }\end{array}$ & $\begin{array}{l}\text { Clinical } \\
\text { success }\end{array}$ & $\begin{array}{l}\text { Procedure } \\
\text { time (min) }\end{array}$ & $\begin{array}{l}\text { Patency } \\
\text { (days) }\end{array}$ & $\begin{array}{l}\text { Dysfunction } \\
\text { (revision) }\end{array}$ & $\begin{array}{l}\text { Survival } \\
\text { (days) }\end{array}$ \\
\hline 1 & $77 / \mathrm{M}$ & $\mathrm{CCC}$ & Advanced cancer & Done & Yes & Yes & 32 & 122 & - & 122 \\
\hline 2 & $92 / F$ & $\mathrm{CCC}$ & Advanced cancer & Done & Yes & Yes & 39 & 109 & - & 109 \\
\hline 3 & $88 / \mathrm{M}$ & $\mathrm{CCC}$ & Advanced LC & None & Yes & None & 31 & 48 & - & 48 \\
\hline 4 & $70 / F$ & $\mathrm{CCC}$ & Advanced cancer & Done & Yes & Yes & 43 & 160 & - & 160 \\
\hline 5 & $69 / M$ & $\mathrm{CCC}$ & Advanced cancer & Done & Yes & Yes & 27 & 177 & $\begin{array}{l}\text { Cholangitis } \\
\text { (none) }\end{array}$ & 198 \\
\hline 6 & $81 / F$ & $\mathrm{CCC}$ & Advanced UC & None & Yes & Yes & 29 & 88 & - & 88 \\
\hline 7 & $74 / \mathrm{M}$ & GC & Advanced GC & Done & Yes & Yes & 32 & 101 & - & 101 \\
\hline
\end{tabular}

\section{Results}

- Table 1 shows patients characteristics. Hybrid metal stent deployment using the novel UCSEMS was attempted in seven patients (median age, 77 years; range, $74-92$ years; male, $n=4$ ). HBO disease was mainly cholangiocarcinoma $(n=6)$. Unresectability largely was a result of advanced stage of cholangiocarcinoma $(n=5)$. Two patients had complications from another advanced carcinoma (lung cancer, $n=1$; uterine cancer, $n=1$ ). In this study, a 8-mm diameter and 8-cm-long UCSEMS was used in all patients. Technical success was obtained in all patients, although mild post-ERCP pancreatitis was seen in one patient, which was successfully treated by conservative treatment. In this study, after stent deployment in the left and posterior bile ducts, a third stent insertion could be performed without dilation of the mesh in the metal stent in the posterior bile duct. Mean procedure time was 33.3 minutes (range, 27 -43). Clinical success was obtained in six patients. On patient in whom clinical success was not achieved received best supportive care. After stent deployment, chemotherapy was attempted in four patients. In this study, stent dysfunction was seen in only one patient. That individual had cholangitis, and because of poor performance status, we treated conservatively. Mean survival in this study was 118 days (range 48 to 198), and duration of stent patency up to patient death was 115 days (range 48 to 177).

\section{Discussion}

Deployment of three UCSEMS is technically challenging. Although the SBS technique is technically easy, deployment of three UCSEMS via SBS may be difficult due to the limited diameter of the biliary tract. Therefore, when three or more UCSEMS are to be deployed, the SIS technique is usually attempted. Kamamoto et al. described the effectiveness of three-branched UCSEMS deployment using the SIS technique in nine consecutive patients [9]. In that study, technical success was obtained in all patients, however, procedure time was relatively long (mean $74.3 \mathrm{~min}$, range, 45-127) compared with our results.
Lee et al. [10] evaluated technical feasibility and efficacy of deployment of a third metal stent to create triple SIS placement in patients with bilateral SIS configuration. Among 156 patients, after stent deployment using the SIS technique, early clinical failure or uncontrolled cholangitis were seen in 21 patients. In addition, insertion of the third stent failed in three patients. Therefore, if guidewire or stent delivery insertion failed after one or two stents deployment using the SIS technique, focal cholangitis of another area can occur. Compared with deployment of a third stent using the SIS technique, the hybrid metal stent technique using the novel UCSEMS has several advantages. First, because the SBS technique is included, deployment of two stents can be performed. Second, compared with the SIS technique, guidewire or stent insertion into the third area is easy. In addition, the shaft of the novel UCSEMS is only 5.4 $\mathrm{Fr}$, and its top is extremely tapered, making stent insertion through the mesh easy compared with experience with the previously described UCSEMS. In addition, we were able to insert all stent delivery systems through the stricture site without pre-dilation. This may be one of the benefits of this stent. However, reintervention may be challenging. In this study, although stent dysfunction was seen in one patient and treated conservatively, reintervention after hybrid stent deployment was not evaluated. Regarding stent patency, the duration of our results is shorter than in with previous reports. This may be based on the short survival in our patient population. Therefore, stent patency after hybrid stent deployment could not be evaluated. These facts are the limitations of this study. In addition, our study has critical limitations such as the small case series, retrospective nature, and single-arm design. A prospective comparison study is needed to verify our results.

\section{Conclusion}

In conclusion, the hybrid metal stent placement technique appears to be useful with the new UCSEMS because of its finegauge stent delivery system. 
Competing interests

None

\section{References}

[1] Lee TH, Kim TH, Moon JH et al. Bilateral versus unilateral placement of metal stents for inoperable high-grade malignant biliary strictures: a multicenter, prospective, randomized study (with video). Gastrointest Endosc 2017; 86: 817-827

[2] Chahal P, Baron TH. Expandable metal stents for endoscopic bilateral stent-within-stent placement for malignant hilar biliary obstruction. Gastrointest Endosc 2010; 71: 195-199

[3] Park DH, Lee SS, Moon JH et al. Newly designed stent for endoscopic bilateral stent-in-stent placement of metallic stents in patients with malignant biliary strictures: multicenter prospective feasibility study (with video). Gastrointest Endosc 2009; 69: 1357-1360

[4] Lee TH, Park do H, Lee SS et al. Technical feasibility and revision efficacy of the sequential deployment of endoscopic bilateral side-by- side metal stents for malignant biliary strictures: a multicenter prospective study. Dig Dis Sci 2013; 58: $547-555$

[5] Inoue T, Okumura F, Naitoh I et al. Feasibility of the placement of a novel 6-mm diameter threaded fully covered self-expandable metal stent for malignant hilar obstructions (with videos). Gastrointest Endosc 2016; 84: $352-357$

[6] Naitoh I, Hayashi K, Nakazawa T et al. Side-by-side versus stent-instent deployment in bilateral endoscopic metal stenting for malignant hilar biliary obstruction. Dig Dis Sci 2012; 57: 3279-3285

[7] Koshitani H, Nakagawa S, Itoh Y. Multiple self-expandable metal stent deployment for unresectable malignant hilar biliary strictures: combination of side-by-side and stent-in-stent methods. Dig Endosc 2016; 28: 621

[8] Cotton PB, Eisen GM, Abakken L et al. A lexicon for endoscopic adverse events: report of an ASGE work shop. Gastrointest Endosc 2010; 71: $446-454$

[9] Kawamoto H, Tsutsumi K, Fujii M et al. Endoscopic 3-branched partial stent-in-stent deployment of metallic stents in high-grade malignant hilar biliary stricture (with videos). Gastrointest Endosc 2007; 66: $1030-1037$

[10] Lee TH, Moon JH, Choi H] et al. Third metal stent for revision of malignant hilar strictures. Endoscopy 2016; 48: 1129-1133 